\title{
A Pilot Split-face Comparison of Q-switched (OS) Single Pulse versus OS Quick Pulse-to-pulse 1,064-nm Nd:YAG Laser Treatment in a Patient with Melasma
}

\author{
Sung Bin $\mathrm{Cho}^{1,2}$ \\ Jin-Soo Kang' \\ Boncheol Leo $\mathrm{Goo}^{3}$
}

${ }^{1}$ Kangskin Clinic, Seoul, Korea
${ }^{2}$ Department of Dermatology and Cutaneous
Biology Research Institute, Yonsei University
College of Medicine, Seoul, Korea
${ }^{3}$ Clinique L Dermatology, Goyang, Korea

Received December 5, 2013

Revised December 23, 2013

Accepted December 24, 2013

\section{Correspondence}

Boncheol Leo Goo

Clinique L Dermatology, 1098, Hangshin-dong,

Goyang 412-220, Korea

Tel: +82-70-4714-6090

Fax: $+82-1600-3660$

E-mail: boncheolgoodgmail.com

(C) Korean Society for Laser Medicine and Surgery

(c) This is an open access article distributed under the terms of the Creative Commons Attribution NonCommercial License (http://creativecommons.org/ licenses/by-nc/3.0) which permits unrestricted noncommercial use, distribution, and reproduction in any medium, provided the original work is properly cited.
Melasma is a common disorder that clinically presents as symmetric illdefined hyperpigmented macules and patches on the face. In the current split-face, evaluator-blinded study, we treated a female patient with eight sessions of 1,064-nm Nd:YAG laser treatment at one-week intervals. Utilizing the $Q$-switched (QS) quick pulse-to-pulse (Q-PTP) mode, in reference to the laser settings, 1,064-nm Nd:YAG laser energy can be irradiated at split fluences and at a dual-pulse interval of $80-\mu \mathrm{sec}$. On the right side of the face, 1,064-nm QS single pulse Nd:YAG laser treatment was administered with the settings of $1.6 \mathrm{~J} / \mathrm{cm}^{2}$, a spot size of 7-mm, and 1200 shots. On the left side, 1,064-nm QS Q-PTP Nd:YAG laser treatment was administered with the settings of $1.6 \mathrm{~J} / \mathrm{cm}^{2}$ irradiated at dual pulses of $0.8 \mathrm{~J} / \mathrm{cm}^{2}$ at $80-\mu \mathrm{sec}$ intervals, a spot size of $7-\mathrm{mm}$, and 1,200 shots. Results of objective clinical assessment showed better clinical outcomes with less treatment-associated pain with QS Q-PTP-treatment than with QS single-pulse-treatment. However, clinical outcomes were subjectively indistinguishable between QS single pulse- and QS Q-PTP-treatments. Transient or persistent post-treatment erythema and newly developed punctate leukoderma lesions were not reported for either side of the face. Pre-existing punctate leukoderma lesions became obscure, especially on the QS Q-PTP-treated side, with improvement of the melasma lesions. We suggest that the QS Q-PTP mode may be of use in treatment of melasma, particularly on the relatively thin skin of the periorbital regions and in pain-sensitive and erythema-prone patients.

\section{Key words}

Nd:YAG laser; Q-switched; Single pulse; Quick pulse-to-pulse; Dual pulse; Split-face; Melasma 


\section{INTRODUCTION}

Melasma is a common disorder that clinically presents as symmetric ill-defined hyperpigmented macules and patches on the face. ${ }^{1}$ Previously, a multicenter survey reported that melasma most commonly affects female patients with Fitzpatrick skin photo types III and IV; reports of melasma lesions are far less common in males than in females. ${ }^{2-4}$

While numerous studies respectively favor the use of various treatment modalities, including topical bleaching agents, chemical peelings, oral tranexamic acid, and several kinds of light or laser devices, the optimal treatment for melasma has not yet been determined and is still under investigation, especially for different skin ethnity. ${ }^{5-8}$ Among the light- or laser-based therapies, use of 1,064-nm Q-switched (QS) Nd:YAG lasers with low fluence for subcellular selective photothermolysis of melanocytes has been effectively and safely applied in the treatment of melasma. ${ }^{5,69-11}$ However, not all patients with melasma achieve satisfactory clinical outcomes with this treatment, and adverse events, including posttreatment petechiae, punctate leukoderma, and rebound hyperpigmentation, commonly develop. ${ }^{12,13}$

In reference to laser settings, the QS quick pulse-topulse (Q-PTP) mode irradiates 1,064-nm Nd:YAG laser energy of split fluence and at dual-pulse intervals of 80- $\mu$ sec. Comparison study between QS single pulse Nd:YAG and QS Q-PTP Nd:YAG treatments, performed on postinflammatory hyperpigmented forearms, demonstrated that QS Q-PTP treatment results in better clinical outcomes without remarkable post-treatment hypopigmentation than QS single pulse treatment. ${ }^{13}$ In the present split-face, evaluator-blinded study, we compared the clinical efficacy and safety of QS single pulse $\mathrm{Nd}$ :YAG versus QS Q-PTP Nd:YAG treatments in a female patient with melasma.

\section{CASE REPORT}

A 33-year-old Korean female patient with Fitzpatrick skin photo type IV participated in this study. This study was reviewed and approved by the ethics board of Clinque L Dermatology (Goyang, Korea) and Lutronic Corporation (Goyang, Korea). The patient was clinically diagnosed with melasma and had previously been treated with topical bleaching agents and laser and light therapies, including 1,064-nm low-fluenced Nd:YAG laser treatment. However, remarkable clinical improvement could not be obtained and the lesions had progressed. She denied having any laser or light treatment or using topical bleaching agents within the previous six months and was not pregnant or immunosuppressed.

After obtaining written informed consent, the patient was treated with eight sessions of 1,064-nm Nd:YAG laser (SPECTRA XT ${ }^{T M}$, Lutronic corporation) treatment at oneweek intervals. On the right side of her face, 1,064-nm QS single pulse Nd:YAG laser treatment was delivered with the settings of $1.6 \mathrm{~J} / \mathrm{cm}^{2}$, a pulse duration of 5-10nsec, a 7-mm spot size, and 1,200 shots with appropriate
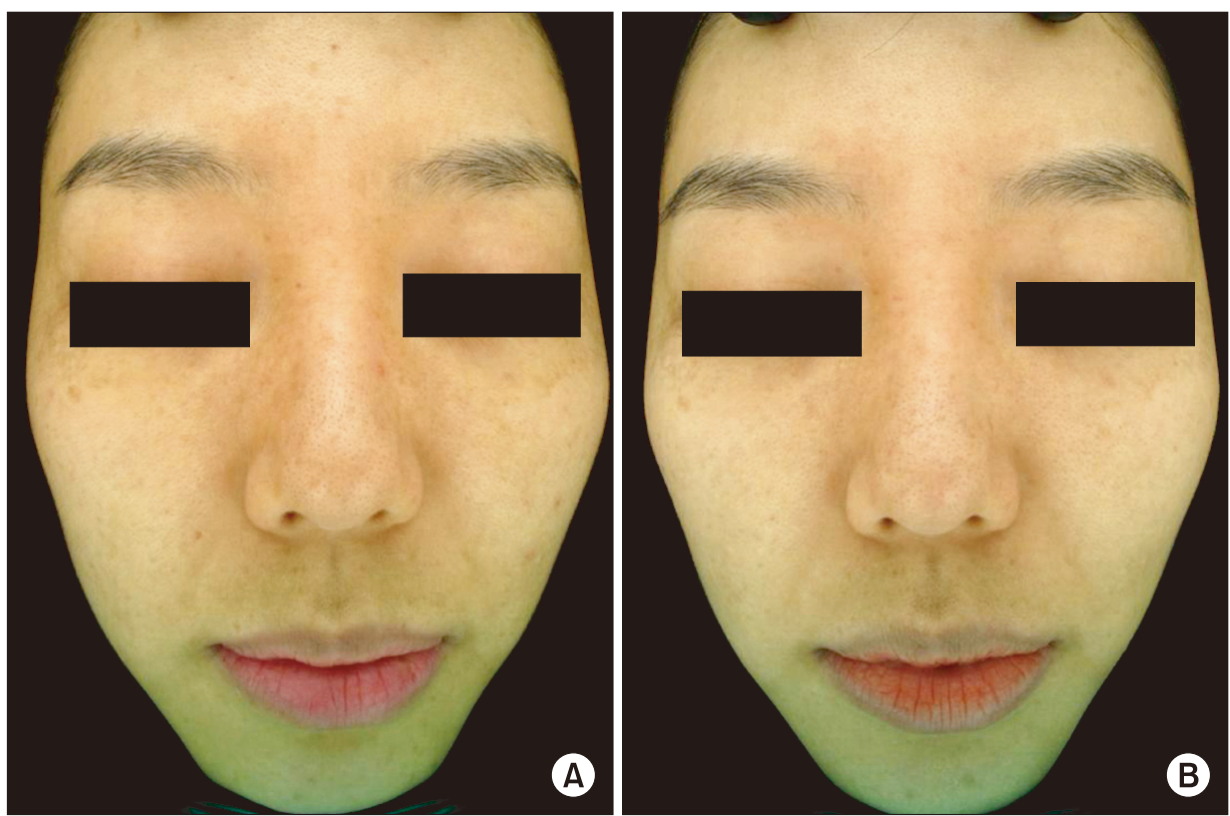

Fig. 1. Normal light-exposed photos show melasma in a 33-year old woman (A) before and (B) one month after eight successive 1,064-nm Nd:YAG laser treatments administered at oneweek intervals. The right side of the face was treated with the Q-switched (QS) single pulse mode, while the left side of the face was treated with the QS quick pulse-to-pulse (Q-PTP) mode. 

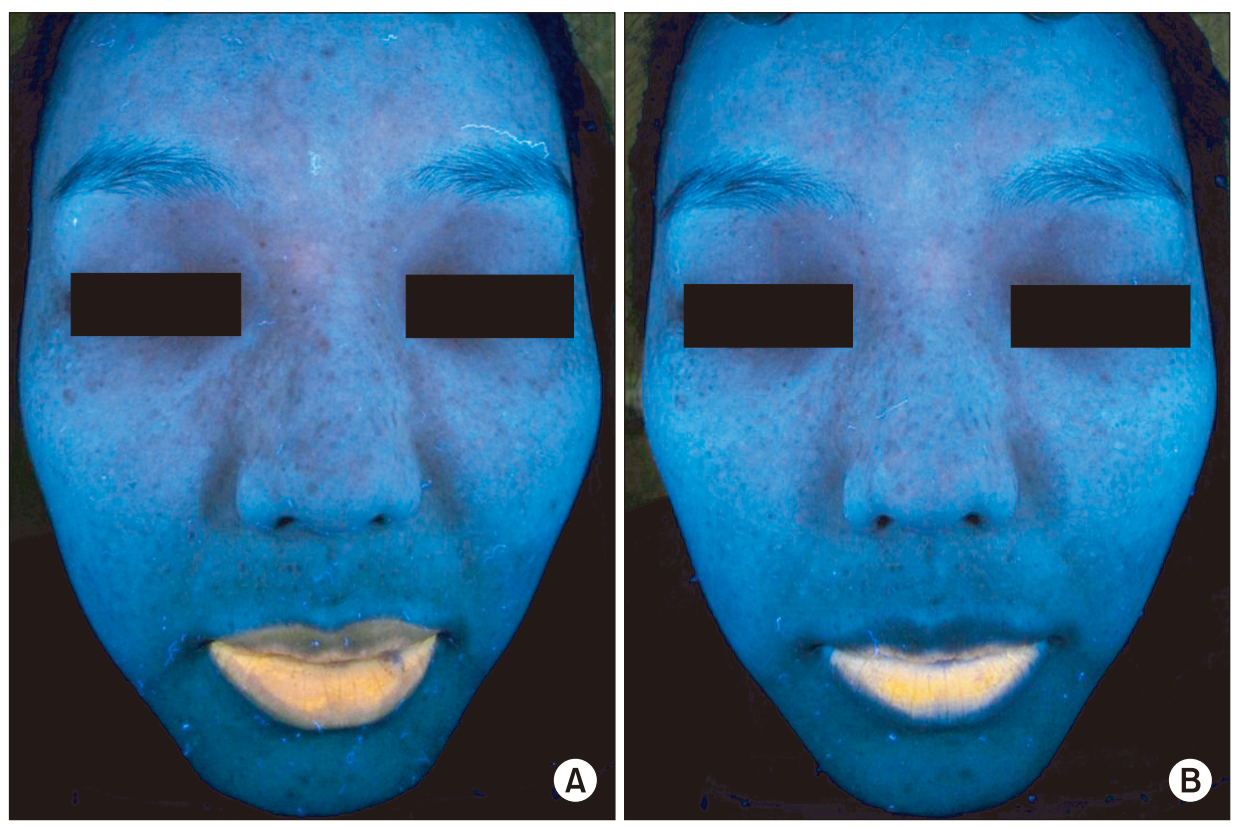

Fig. 2. Ultraviolet light-exposed photos taken (A) before and (B) one month after eight sessions of 1,064$\mathrm{nm} \mathrm{Nd}$ :YAG laser treatment show hyperpigmented patches distributed across the patient's forehead, perioral area, and nose, as well as malar areas.
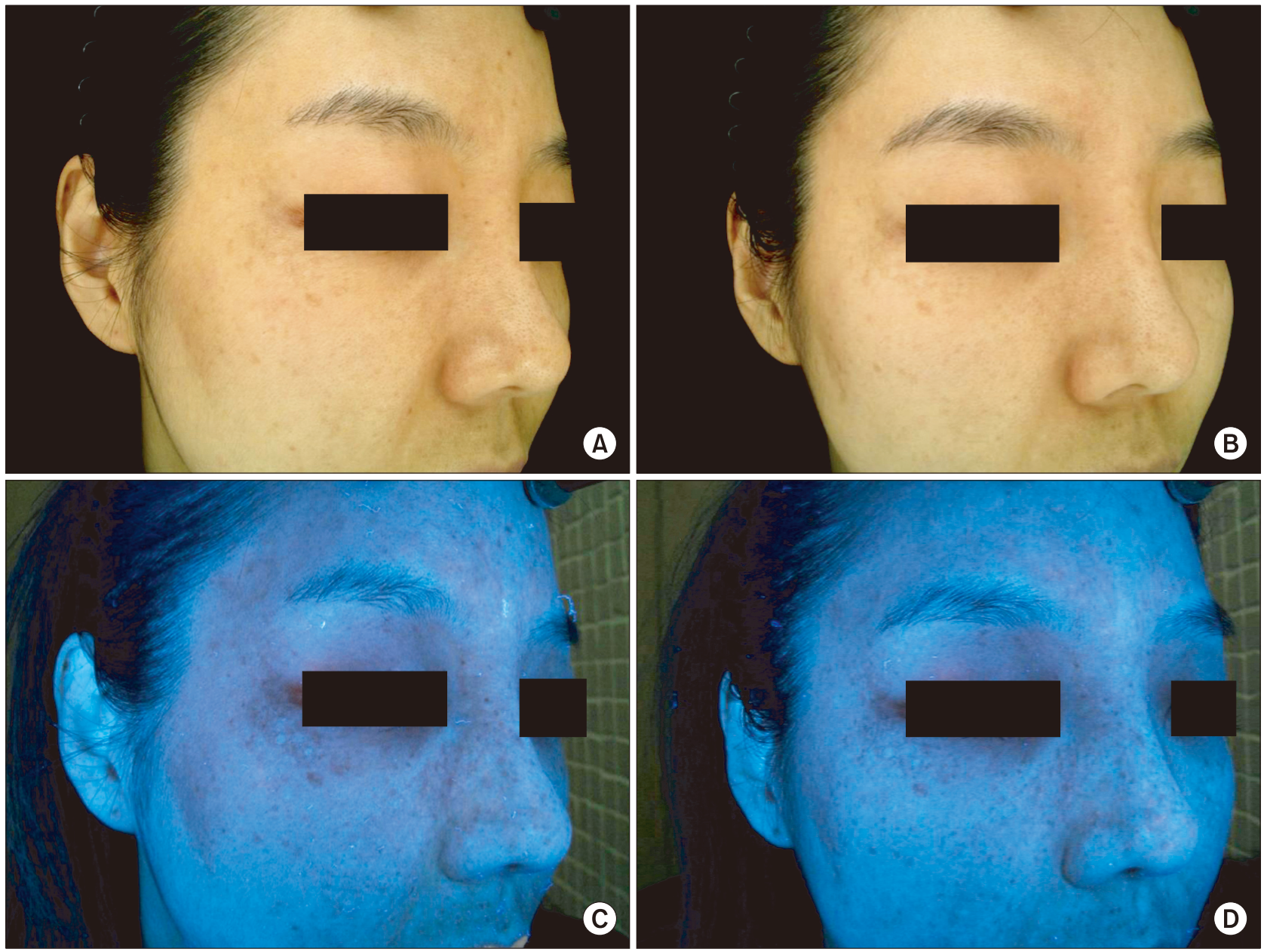

Fig. 3. (A, B) Normal light-exposed and (C, D) ultraviolet-violet exposed photos of the right side of the face treated with 1,064-nm QS single pulse Nd:YAG laser treatment. (A and C) Before and (B and D) one month after the laser treatments. 
overlapping. On the left side of her face, 1,064-nm QS Q-PTP Nd:YAG laser treatment was delivered with the settings of $1.6 \mathrm{~J} / \mathrm{cm}^{2}$, irradiated at dual pulses of $0.8 \mathrm{~J} /$ $\mathrm{cm}^{2}$ and $80-\mu \mathrm{sec}$ intervals, a pulse duration of 5-10nsec, a $7-m m$ spot size, and 1200 shots with appropriate overlapping. During the course of treatment, she used neither bleaching agents nor oral contraceptives. The patient was recommended to avoid excessive sunexposure and to apply a broad-spectrum sunscreen during and after the treatment, equally on both side of her face.

Objective clinical assessment was performed at one month after the final treatment by three separate blinded dermatologists using photographs taken before and after each treatment with a pigment imaging tool (Janus ${ }^{\circledR}$, PSI corporation, Ltd., Seoul, Koreal under normal and ultraviolet light exposures. Objective improvement score was also recorded using a global aesthetic improvement scale (GAIS) of 'worse than before' (score: -1); 'clinically unchanged' (score: 0); 'slightly improved' (score: 1); 'moderately improved' (score: 2); and 'markedly improved' (score: 3). The patient was also asked to report on pain during the treatment using a visual analogue scale (VAS) and any side effects.

Initial photos under normal light exposure showed malar distribution of melasma lesions (Fig. 1A). Additional photos taken under ultraviolet light exposure revealed hyperpigmented patches distributed along the patient's forehead, perioral area, and nose, as well as malar areas (Fig. 2A). Overall, clinical improvement was noticeable from the third session of QS single pulse and QS Q-PTP 1,064-nm Nd:YAG laser treatments, and remarkable improvement was recorded at one month after final treatment (Fig. 1B and 2B).

Objective clinical assessment revealed better clinical outcomes (mean GAIS score: 2.7 in QS Q-PTP vs. 2.3 in
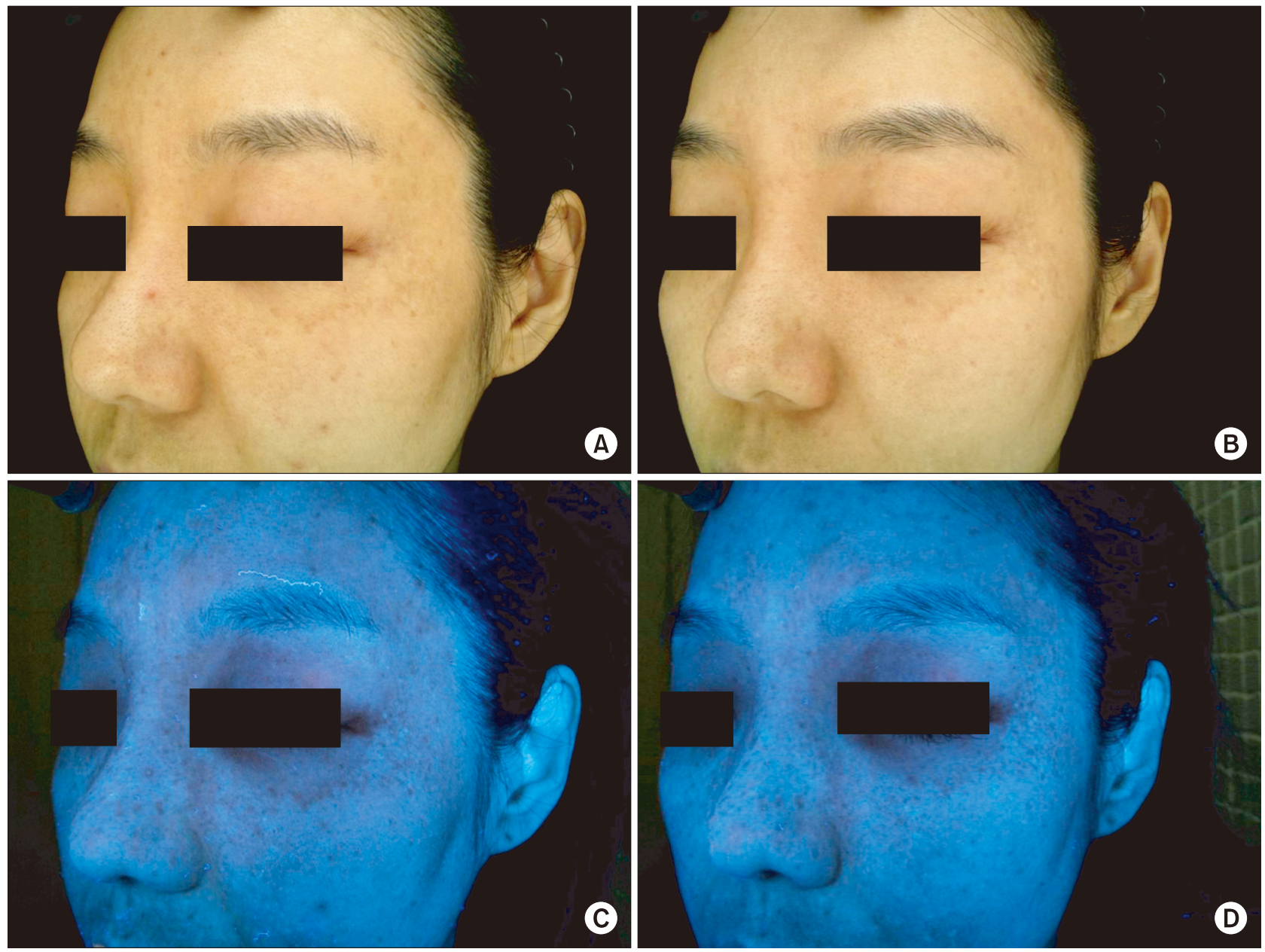

Fig. 4. (A, B) Normal light-exposed and (C, D) ultraviolet-violet exposed photos of the left side of the face treated with 1,064-nm QS Q-PTP Nd:YAG laser treatment. (A and C) Before and (B and D) one month after the laser treatments. 
QS single pulse) with less treatment-associated pain (VAS score: 2 in QS Q-PTP vs. 4 in QS single pulse) with the QS Q-PTP-treatment than the QS single pulse-treatment (Fig. 3 and 4). Nevertheless, the patient was satisfied with the clinical outcomes on both sides of her face. Moreover, while lesser treatment-associated pain was reported for the QS Q-PTP-treated side, clinical outcomes were subjectively indistinguishable between QS single pulseand QS Q-PTP-treatments. Transient or persistent posttreatment erythema was not recorded for either side of the face. Pre-existing punctate leukoderma lesions, which were noticed on the ultraviolet light-exposed baseline photos, became obscure, especially on the QS Q-PTP-treated side, with improvement of the melasma lesions. Also, on either side, no newly developed punctate leukoderma lesions were observed.

\section{DISCUSSION}

The use of a 1,064-nm QS Nd:YAG laser of low fluence and $5-10-n s e c$ pulse duration has been investigated by numerous studies for the treatment of melasma. 5,6,9,14,15 After application of such treatment, the ultrastructural features of epidermal melanocytes have been shown to comprise fewer dendrites. ${ }^{14}$ Additionally, according to a transmission electron microscopic study, mature stage IV melanosomes can be selectively photothermolysed using a low-fluenced QS Nd:YAG laser. ${ }^{14}$ Moreover, preservation of epidermal melanocytes can be achieved with the use of low fluence nonablative laser treatment with a short pulse duration for selective photothermolysis of target melanosomes. $6,9,11,14$

Previously, histopathological analyses of patients with melasma, who demonstrated remarkable clinical improvement after successive treatment of low fluence 1,064-nm QS Nd:YAG laser, revealed significant decreases in the expression of melanogenesis-associated proteins, including tyrosinase-related proteins, $\alpha$ melanocytic-stimulating hormone, stem cell factor, and nerve growth factor, after laser treatment. ${ }^{15}$ However, Melan-A and SOX-10 expression was not affected by the laser treatment. ${ }^{15}$ Post-laser treatment punctate leukoderma, an adverse event associated with relatively higher fluence in this kind of 1,064-nm QS Nd:YAG laser treatment approach, also presented the normal number of melanocytes, but reduced the number and size of melanosomes. $^{15}$

In the present study, we compared the therapeutic efficacy and safety of QS single pulse mode and QS Q-PTP mode laser treatment of melasma, utilizing a 1,064-nm
$\mathrm{Nd}: Y A G$ laser. We used the laser settings of $1.6 \mathrm{~J} / \mathrm{cm}^{2}$ and a 7-mm spot size, and evaluated the patient with photos under normal and ultraviolet light exposure as described in the previous report. ${ }^{16}$ In doing so, we recorded remarkable clinical outcomes for QS Q-PTP mode laser treatment that were similar to those for QS single pulse mode laser treatment, even with the half-fluence and dual-pulse treatment at $80-\mu$ sec intervals. Although only one subject was investigated in this pilot study, the result exhibited better objective improvements in the refractory melasma lesions along the malar area on the QS Q-PTPtreated side of the face than on the QS single pulsetreated side. In our study, we used a 1,064-nm Nd:YAG laser with 80-usec pulse intervals, which were shorter than those in a previous report. ${ }^{13}$ We discerned that laser pulses could have delivered on the approximately identical target chromophores by shortening the intervals between dual pulses.

The patient in our study initially presented with postlaser treatment punctate leukoderma on the melasma lesions, from previous treatments. A previous report suggested that the risk factors for this adverse event include the use of a relatively high-energy laser setting, non-uniform energy output of QS Nd:YAG laser, the delivery of multiple passes, and treatment of melasma lesions on periorbital areas. ${ }^{15,16}$ As well, patients who present with post-laser therapy transient erythema are known to be at risk for developing hypopigmented macules within the melasma lesions. ${ }^{15}$ Notwithstanding, in the present study, with use of the QS Q-PTP mode, preexisting post-laser treatment punctate leukoderma became noticeably obscure, even though the melasma lesions were located along periorbital areas in our erythema-prone patient.

In this split-face, evaluator-blinded study, QS Q-PTP $\mathrm{Nd}$ :YAG treatment was proved to be safe and effective in treating periorbital melasma in a single female patient. Accordingly, we suggest that the QS Q-PTP mode may be of use in treating melasma, especially on the relatively thin skin of the periorbital regions and in pain-sensitive and erythema-prone patients.

\section{REFERENCES}

1. Grimes PE. Melasma. Etiologic and therapeutic considerations. Arch Dermatol 1995;131:1453-7.

2. Ortonne JP, Arellano I, Berneburg M, Cestari T, Chan H, Grimes $P$, et al. A global survey of the role of ultraviolet radiation and hormonal influences in the development of melasma. J Eur Acad Dermatol Venereol 2009;23:1254-62. 
3. Kang WH, Yoon KH, Lee ES, Kim J, Lee KB, Yim H, et al. Melasma: histopathological characteristics in 56 Korean patients. Br J Dermatol 2002;146:228-37.

4. Sheth VM, Pandya AG. Melasma: a comprehensive update: part I. J Am Acad Dermatol 2011;65:689-97.

5. Cho SB, Kim JS, Kim MJ. Melasma treatment in Korean women using a 1064-nm Q-switched Nd:YAG laser with low pulse energy. Clin Exp Dermatol 2009;34:e847-50.

6. Shin JU, Park J, Oh SH, Lee JH. Oral tranexamic acid enhances the efficacy of low-fluence 1064-nm quality-switched neodymiumdoped yttrium aluminum garnet laser treatment for melasma in Koreans: a randomized, prospective trial. Dermatol Surg 2013; 39:435-42.

7. Taylor CR, Anderson RR. Ineffective treatment of refractory melasma and postinflammatory hyperpigmentation by Qswitched ruby laser. J Dermatol Surg Oncol 1994;20:592-7.

8. Stratigos AJ, Dover JS, Arndt KA. Laser treatment of pigmented lesions--2000: how far have we gone? Arch Dermatol 2000;136: 915-21.

9. Suh KS, Sung JY, Roh HJ, Jeon YS, Kim YC, Kim ST. Efficacy of the 1064-nm Q-switched Nd:YAG laser in melasma. J Dermatolog Treat 2011;22:233-8.

10. Zhou X, Gold MH, Lu Z, Li Y. Efficacy and safety of Q-switched 1,064-nm neodymium-doped yttrium aluminum garnet laser treatment of melasma. Dermatol Surg 2011;37:962-70.
11. Kim JH, Kim H, Park HC, Kim IH. Subcellular selective photothermolysis of melanosomes in adult zebrafish skin following 1064-nm Q-switched Nd:YAG laser irradiation. J Invest Dermatol 2010;130:2333-5.

12. Kim MJ, Kim JS, Cho SB. Punctate leucoderma after melasma treatment using 1064-nm Q-switched Nd:YAG laser with low pulse energy. J Eur Acad Dermatol Venereol 2009;23:960-2.

13. Kim BW, Lee MH, Chang SE, Yun WJ, Won CH, Lee MW, et al. Clinical efficacy of the dual-pulsed $\mathrm{Q}$-switched neodymium: yttrium-aluminum-garnet laser: Comparison with conservative mode. J Cosmet Laser Ther 2013;15:340-1.

14. Mun JY, Jeong SY, Kim JH, Han SS, Kim IH. A low fluence Q-switched Nd:YAG laser modifies the 3D structure of melanocyte and ultrastructure of melanosome by subcellularselective photothermolysis. J Electron Microsc (Tokyo) 2011;60:11-8.

15. Kim JE, Chang SE, Yeo UC, Haw S, Kim IH. Histopathological study of the treatment of melasma lesions using a low-fluence Q-switched 1064-nm neodymium:yttrium-aluminium-garnet laser. Clin Exp Dermatol 2013;38:167-71.

16. Chan NP, Ho SG, Shek SY, Yeung CK, Chan HH. A case series of facial depigmentation associated with low fluence $Q$-switched 1,064 nm Nd:YAG laser for skin rejuvenation and melasma. Lasers Surg Med 2010;42:712-9. 